\title{
Desarrollo renal fetal alterado y riesgo aumentado de enfermedades del adulto
} Alterations in fetal kidney development and increased risk for
adult diseases

\author{
Dr. Carlos Grandia,b
}

\section{RESUMEN}

El bajo peso al nacer (BP, $<2500 \mathrm{~g})$, la restricción del crecimiento intrauterino (RCIU) y el parto prematuro (PP, < 37 semanas de gestación) son los factores clínicos más habituales para la programación alterada del número de nefronas y se asocian con un mayor riesgo de hipertensión, proteinuria y enfermedad renal futura en la vida. En la actualidad la evaluación indirecta del número total de nefronas mediante el uso de marcadores en el período posnatal representa el enfoque principal para evaluar el riesgo de evolución futura de los trastornos renales en los recién nacidos con $\mathrm{BP}$, RCIU o PP.

Se presentan los avances en la investigación en animales y sobre marcadores bioquímicos en humanos, y recomendaciones para la prevención del daño renal preconcepcional, incluidos los factores sociales y las enfermedades crónicas. La evidencia demuestra que la restricción de crecimiento y la prematuridad solas son capaces de modular la nefrogénesis y la función renal y, cuando son concurrentes, sus efectos tienden a ser acumulativos.

Palabras clave: nefronas, enfermedades renales, recién nacido de bajo peso, restricción del crecimiento

a. Subcomisión Origen y Enfermedad en el Curso de la Vida (DOHaD),

Sociedad Argentina de Pediatría, Ciudad Autónoma de Buenos Aires,

Argentina.

b. Departamento de Pediatría, Facultad de Medicina de Ribeirão Preto, Universidad de San Pablo, Brasil.

Correspondencia: Dr. Carlos Grandi: cgrandi@intramed.net

Financiamiento:

Ninguno.

Conflicto de intereses:

Ninguno que declarar.

Recibido: 4-12-2020

Aceptado: 27-4-2021

\section{INTRODUCCIÓN}

El enfoque del curso de la vida se alinea con los objetivos propuestos por la Agenda 2030 para el Desarrollo Sostenible de las Naciones Unidas, con las recomendaciones de la Organización Mundial de la Salud en la Declaración de Minsk y el Plan de Acción Mundial para la Prevención y el Control de Enfermedades Crónicas no Transmisibles (ECNT).

Se sugiere un enfoque mucho más amplio para mantener la salud, que incluya el aspecto del desarrollo renal y prevenir o evitar la enfermedad renal. ${ }^{1,2}$

\section{INFLUENCIAS AMBIENTALES}

Estudios epidemiológicos demostraron que la etapa previa a la concepción, la fetal y la perinatal pueden contribuir al desarrollo de enfermedades renales del adulto (Figura).

Un metaanálisis de 31 estudios, que incluyó a más de 2 millones de sujetos, documentó que los niños con $\mathrm{BP}$ al nacer tuvieron un riesgo del $70 \%$ de desarrollar enfermedad renal crónica (ERC) en la edad adulta, definida como albuminuria, reducción del filtrado glomerular o insuficiencia renal. ${ }^{3}$ Aunque los cambios en la función renal son generalmente leves e incluso pueden estar dentro del rango normal en niños y adolescentes, estos pueden progresar a disfunción renal manifiesta con la edad o a lesiones renales superpuestas.

La exposición a diabetes, sobrepeso y obesidad gestacional, así como una nutrición materna por debajo de los niveles óptimos, se reconocen cada vez más como factores de riesgo para 
la programación temprana del desarrollo renal en la descendencia. Estos datos se basan en el hallazgo de que la reserva funcional renal es menor en los hijos adultos jóvenes de madres con diabetes durante la gestación que en aquellos con padres diabéticos. ${ }^{4,5}$

Esta relación se ve amplificada por el desarrollo de lesión renal aguda en los recién nacidos prematuros, que puede reducir aún más el número de nefronas poco después del nacimiento. También un rápido crecimiento de recuperación (crecimiento compensatorio o catchup) o sobrealimentación durante la infancia en niños nacidos pequeños puede aumentar aún más el riesgo de hipertensión, ERC y predisponer a la obesidad y la diabetes de tipo 2 a lo largo de la vida. ${ }^{3}$

Los medicamentos administrados a las madres antes del parto también se asociaron con un impacto sobre la función renal neonatal. La terapia tocolítica prenatal administrada a la madre se vinculó significativamente con una tasa de filtración glomerular (TFG) más baja en el lactante de 7 días. ${ }^{6}$ Otros informes sugieren que la administración de la nimesulida, un inhibidor de la COX-2, como tocolítico induce insuficiencia y enfermedad renal crónica en neonatos. ${ }^{7}$

Estudios recientes han identificado mutaciones en el gen APOL1 que están asociadas con un mayor riesgo de glomeruloesclerosis segmentaria focal entre individuos de ascendencia africana; sin embargo, los estudios a gran escala del genoma no han podido identificar genes dominantes o polimorfismos asociados con la mayoría de las enfermedades renales. ${ }^{8}$ En contraste con los paradigmas genéticos y de estilo de vida como determinantes de las ECNT, el paradigma del desarrollo de la salud y la enfermedad (DOHaD) ha surgido como un modelo fisiopatológico complementario y más integral del riesgo de ECNT.

FIGURA. Origen de múltiples condiciones en la programación de la enfermedad renal

EMBARAZO

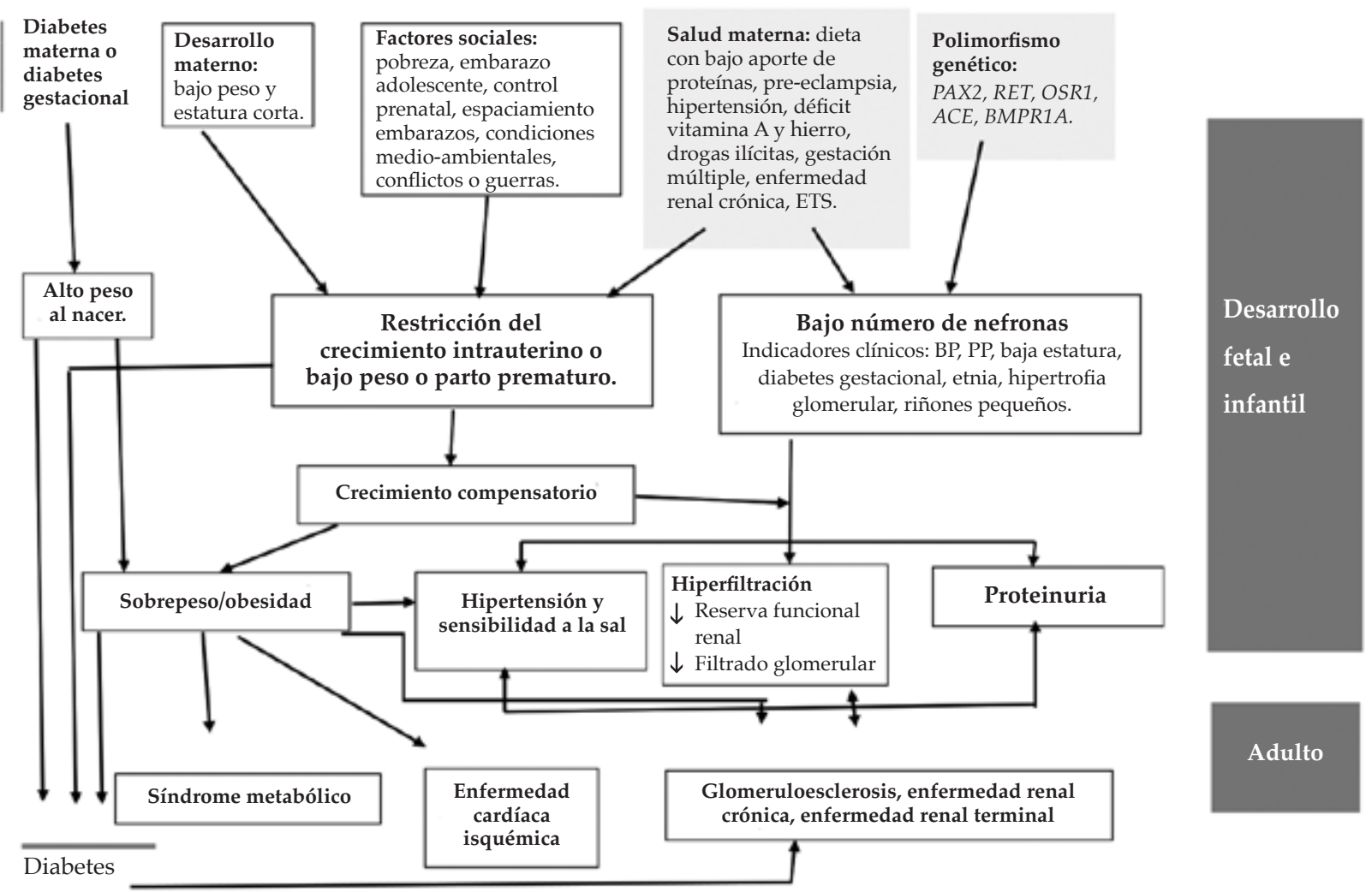

BP: bajo peso ( $<2500$ g); PP: parto prematuro (<37 semanas de edad gestacional), ETS: enfermedades de transmisión sexual). Modificado de Luyckx et al. ${ }^{12}$ 


\section{PROGRAMACIÓN DEL DESARROLLO DEL NÚMERO DE NEFRONAS}

En condiciones normales de desarrollo, la nefrogénesis continúa hasta la 36 . $^{a}$ semana de gestación y no se desarrollan nuevas nefronas después del nacimiento a término. ${ }^{9}$ En los recién nacidos prematuros, la nefrogénesis puede ocurrir hasta 40 días después del nacimiento, pero puede ser anormal en prematuros extremos. ${ }^{10,11}$

La programación alterada del número de nefronas es un nexo importante entre la exposición a factores estresantes del desarrollo y el riesgo posterior de hipertensión arterial y enfermedad renal. ${ }^{12,13} \mathrm{El} \mathrm{BP}$ al nacer, la RCIU y el PP son los factores clínicos más habituales para el bajo número de nefronas y se asocian con un mayor riesgo de hipertensión, proteinuria y enfermedad renal más tarde en la vida. ${ }^{12}$

Los datos de riñones humanos examinados post mortem muestran una correlación directa entre el número de nefronas y el peso al nacer en todo el espectro de edad del paciente. A partir de estos datos, se infiere un aumento de 257426 glomérulos por kilogramo de aumento en el peso al nacer. ${ }^{14}$

El consenso general es que existen aproximadamente un millón de nefronas por riñón; sin embargo, los datos actuales indican que el número de nefronas varía notablemente entre individuos, hasta 13 veces en algunas poblaciones. Una gran proporción de variabilidad en el número de nefronas ya es evidente al nacer, lo que sugiere un fuerte efecto en el desarrollo. ${ }^{14}$ Individuos nacidos con el número total de nefronas hacia el extremo inferior del rango pueden ser más propensos a desarrollar hipertensión y disfunción renal, especialmente después de la exposición posterior a estrés renal, como pérdida adicional de nefronas, hipertensión o diabetes. ${ }^{15}$

Sobre la base de evidencia epidemiológica temprana que vincula el BP y la enfermedad cardiovascular de adultos, Brenner et al., ${ }^{16}$ plantearon la hipótesis de que la programación del desarrollo en el riñón puede resultar en una reducción en el número de nefronas, lo que a su vez puede ser un factor que contribuye a una presión arterial más alta y un mayor riesgo de ERC.

\section{EVALUACIÓN INDIRECTA DEL NÚMERO TOTAL DE NEFRONAS}

Hasta la fecha, la evaluación indirecta del número total de nefronas usando marcadores clínicos, de imágenes, bioquímicos y moleculares en el período posnatal representa el enfoque principal para evaluar el riesgo de evolución futura de los trastornos renales en los recién nacidos con $\mathrm{BP}$ al nacer. ${ }^{17}$

El número de nefronas es una variable crítica en la defectuosa maduración renal y progresión a enfermedad renal. En consecuencia, es un indicador importante de la capacidad funcional del riñón, y un número bajo de nefronas se considera un factor de riesgo significativo para la enfermedad renal. ${ }^{18-20}$

La reducción de la nefrogénesis puede estar asociada a hiperfiltración, hipertensión, daño glomerular y proteinuria, lo que a su vez inicia un ciclo vicioso que conduce a la pérdida de unidades funcionales y a la insuficiencia renal a largo plazo. ${ }^{21}$ La enfermedad renal es, por lo tanto, una prioridad mundial de salud pública. Dado el costo elevado del tratamiento, tanto para el individuo como para la sociedad, la prevención es la estrategia más efectiva para abordar de manera sostenible la creciente carga mundial de la enfermedad renal.

\section{INVESTIGACIÓN EN ANIMALES Y MARCADORES BIOQUÍMICOS EN HUMANOS}

Los avances en la investigación en animales y sobre marcadores bioquímicos en humanos motivaron un consenso que resalta la necesidad de actuar temprano para prevenir la ERC y otras enfermedades no transmisibles relacionadas más tarde en la vida, asociadas al bajo número de nefronas al nacimiento con $\mathrm{BP}$, tamaño pequeño para la edad gestacional (PEG) y prematuridad, y de diseñar intervenciones coordinadas. ${ }^{22}$ Estas estrategias permitirán optimizar intervenciones para limitar o interrumpir el ciclo de programación del desarrollo de la ERC a lo largo de la vida, especialmente en las regiones más subdesarrolladas del mundo.

\section{EXPERIMENTACIÓN EN ANIMALES}

El objetivo del estudio de Myrie et al., ${ }^{23}$ novedoso modelo de RCIU espontáneo en cerdos en miniatura de Yucatán, permitió evaluar la asociación del deterioro del crecimiento fetal y el rápido crecimiento posnatal con la programación de la hipertensión y el síndrome metabólico en la edad adulta. A los 9 meses de edad, se implantaron telémetros de presión arterial (PA) para evaluar la PA antes $(0,5 \%$ de $\mathrm{ClNa})$ y después $(4,5 \%$ de $\mathrm{ClNa})$ de un período de carga de sal de 7 días. A los 10 meses de edad se 
determinó el número de nefronas. Los cerdos con RCIU mostraron una ingesta relativamente mayor de ClNa $(p<0,05)$ y experimentaron un catchup significativo. Los cerdos adultos con RCIU también tuvieron mayor PA y un $43 \%$ menos de nefronas por riñón $(p<0,05)$. El número de nefronas se asoció positivamente con el peso al nacer y presentó una correlación negativa con la PA $(p<0,05)$.

El estudio de Boubred et al., ${ }^{24}$ demostró que la hipertensión arterial y la ERC inducidas por la RCIU están vinculadas a la magnitud de la reducción del número de nefronas, independientemente de su causa. La presión arterial sistólica, la TFG, la proteinuria, el número de nefronas y la esclerosis glomerular se compararon entre crías de ratas con RCIU expuestas prenatalmente a una dieta materna baja en proteínas (9\% de caseína; descendencia LPD) o administración materna de betametasona (descendencia BET). Ambas intervenciones prenatales llevaron al RCIU y una reducción similar en el peso al nacer. En comparación con los controles normales, la descendencia BET tuvo un grave déficit de nefronas $(-50 \%, p<0,01)$, una TFG deteriorada $(-33 \%, p<0,05)$ e hipertensión arterial sistólica $(+17 \mathrm{mmHg}, p<0,05)$. La esclerosis glomerular fue más del doble en la descendencia BET que en la descendencia control $(p<0,05)$. Las funciones y la estructura renales no se modificaron en las crías de LPD a largo plazo, si bien tenían un déficit moderado de nefronas en comparación con las BET. Los mecanismos propuestos por los cuales las manipulaciones dietéticas influyen en el número de nefronas incluyen la modificación epigenética, la modulación global de la expresión génica, la mayor exposición fetal a los glucocorticoides debido a la disminución en la expresión de la enzima $11 \beta$-dehidroxiesteroide deshidrogenasa 2 (11ßHSD2) placentaria que metaboliza los glucocorticoides maternos y evita que lleguen al feto, y disminuya la angiogénesis renal..$^{25,26}$

Los estudios que utilizan modelos de ratas demostraron que el número de nefronas se reduce en la descendencia en proporción a los niveles maternos de vitamina A. Una reducción en el número de nefronas podría estar mediada por los efectos del ácido transretinoico en la ramificación de los brotes ureterales y el crecimiento vascular en el riñón en desarrollo. ${ }^{27}$ La provisión de una dieta posnatal óptima, en ratas con $\mathrm{BP}$ al nacer, cuando la nefrogénesis está en curso, condujo a la restauración del número de nefronas y a la prevención de la hipertensión subsiguiente. ${ }^{28}$ Una revisión sistemática confirmó que las deficiencias de folato materno, vitamina A y aporte energético total durante el embarazo se asociaron con efectos perjudiciales en la estructura y función renal. ${ }^{5}$

Estudios recientes que utilizan resonancia magnética con ferritina pueden medir con precisión el número glomerular en ratones y se han utilizado ex vivo en riñones humanos; sin embargo, estas técnicas aún están lejos de ser clínicamente aplicables de manera no invasiva. ${ }^{22,29}$

Estos hallazgos resaltan la importancia de desarrollar marcadores tempranos del número de nefronas y la necesidad de un seguimiento a largo plazo de los adultos que fueron prematuros expuestos a los glucocorticoides en el período prenatal o posnatal.

\section{MARCADORES SUSTITUTOS DEL NÚMERO DE NEFRONAS}

Tanto la prematuridad como el RCIU se asocian con un mayor riesgo de desarrollar lesiones renales en la vida adulta. La evidencia sostiene que, una vez que se establece el deterioro funcional renal, a menudo es difícil retrasar la evolución de la enfermedad..$^{30}$ En consecuencia, es crucial identificar lo antes posible a aquellos prematuros y a los recién nacidos con RCIU con riesgo de desarrollar enfermedad renal para iniciar medidas preventivas antes de que se establezca la disfunción renal. ${ }^{31}$

Actualmente, la evaluación indirecta del número total de nefronas mediante el uso de marcadores en el período posnatal representa el enfoque principal para evaluar el riesgo de evolución futura de los trastornos renales en los recién nacidos con BP. ${ }^{32}$ De hecho, se cree que la reducción del número de nefronas, por disminución de la nefrogénesis, es una causa importante de evolución renal adversa en este grupo..$^{18}$

El estudio de Aisa et al., ${ }^{33}$ determinó la posible aparición temprana de daño renal en niños con RCIU y en recién nacidos prematuros a los 30 a 40 días de la edad posnatal corregida mediante la medición de varios indicadores urinarios de daño y/o lesión glomerular y tubular, como el transporte de sodio, el sistema renina-angiotensina-aldosterona y la actividad simpática, que regulan la función renal. Como los glomérulos constituyen el $95 \%$ del volumen cortical renal, en ausencia de una aparente hipertrofia glomerular inducible en esta fase posnatal, se considera que el volumen cortical renal es indicativo del número de nefronas. ${ }^{34}$ 
Se evaluaron los niveles de albúmina, la actividad de $\mathrm{N}$-acetil- $\beta$-D- glucosaminidasa (NAG) y la catepsina $B$ en neonatos prematuros y con RCIU a una edad corregida de 30 a 40 días, y se los comparó con los datos de una población de recién nacidos sanos a término. Específicamente, el nivel de excreción de proteinuria puede relacionarse tanto con la función glomerular como con la tubular, mientras que la albuminuria es un marcador de la permeabilidad glomerular. La NAG es un marcador temprano, sensible, persistente y robusto de lesión y / o disfunción del túbulo proximal. ${ }^{35} \mathrm{El}$ volumen renal total y el volumen cortical se reconstruyeron y se estimaron utilizando la tecnología de ecografía 3-D combinada con el análisis asistido por ordenador de órganos virtuales, una tecnología altamente reproducible y precisa para la evaluación de los volúmenes de órganos. ${ }^{36,37}$

Se observaron niveles bastante mayores de todos los parámetros bioquímicos probados en los neonatos con RCIU, mientras que en los recién nacidos prematuros se observó un aumento significativo en la actividad de la catepsina B, el nivel de proteinuria $\mathrm{y}$, en menor medida, el nivel de albuminuria. La actividad de catepsina B mostró una correlación significativa, fuerte e inversa con todos los marcadores sustitutos del número de nefronas y también se correlacionó de manera fuerte y positiva con el nivel de albuminuria. ${ }^{36,37}$

El volumen renal total y el volumen cortical fueron significativamente más bajos en los neonatos con RCIU y PP que en los neonatos de control. Además, la proteinuria y los niveles de albuminuria, y la actividad de NAG y de catepsina $B$ se asociaron inversamente con todos los marcadores indirectos del número de nefronas, como la edad gestacional, peso al nacer, volumen renal total y volumen cortical. ${ }^{37}$

El menor número de nefronas en los neonatos de BP al nacer se vinculó a lesión y / o deterioro tubular que podría coexistir con una disfunción de la permeabilidad glomerular. Datos recientes indican que, luego de insuficiencia úteroplacentaria, los riñones de ratas con RCIU se caracterizaron por una expresión alterada de los genes implicados en el desarrollo y función tubular, tanto al nacer como en el período posnatal temprano. ${ }^{38}$

Estos datos demuestran que tanto el RCIU como la prematuridad por separado son capaces de modular la nefrogénesis y la función renal y que, cuando son concurrentes, sus efectos tienden a ser acumulativos.

\section{RECOMENDACIONES PARA LA PREVENCIÓN DEL DAÑO PRECONCEPTIVO}

Los efectos transgeneracionales de la programación del desarrollo sugieren que la atención temprana para reducir el riesgo de enfermedad a largo plazo producirá el mayor beneficio para el individuo y para las generaciones posteriores.

La etapa preconcepcional es un período crítico para desarrollar intervenciones adecuadas para asegurar la salud tanto materna como paterna y así prevenir la potencial aparición de las ECNT en la vida adulta:

1. Implementar programas integrales preconcepcionales para la atención general y especializada, y educación sexual de niñas en edad escolar primaria y secundaria, ya que este período es uno de los más críticos en el curso de la vida.

2. Brindar asesoramiento previo a la concepción con respecto a modificación de la dieta, control de peso, ejercicio físico y opciones de estilo de vida para optimizar la salud materna a futuro y la evolución del recién nacido.

3. Identificar y tratar enfermedades y sus complicaciones, tales como diabetes preexistente, insuficiencia renal, hipertensión, anemia e infecciones, que pueden afectar la salud materna, fetal y neonatal.

4. Implementar atención preconcepcional de rutina, atención de forma inmediata en el período posparto después de cada parto o pérdida del embarazo, y atención interconcepcional.

5. Las instituciones y los Gobiernos deben implementar medidas no médicas de control de salud. Con respecto a los factores de riesgo relacionados con la pobreza, algunas podrían incluir la reducción de embarazos adolescentes, el acceso a la educación sexual integral para niños, niñas y adolescentes, para asegurar el acceso a la planificación familiar, espaciar los embarazos y evitar experiencias de violencia.

Estas recomendaciones resaltan la necesidad de actuar tempranamente para reducir el BPN, PEG, la prematuridad y el bajo número de nefronas al nacer, con el objetivo de prevenir la ERC y ECNT en el curso de la vida a través de la intervención coordinada de obstetras, neonatólogos, nefrólogos y médicos de familia. ${ }^{11,22,39}$

Una nueva propuesta es el abordaje preventivo, que comienza en el período 
perinatal en recién nacidos prematuros y de $\mathrm{BP}$, mediante la inducción de células madre renales pluripotenciales para generar nuevas nefronas después del nacimiento, para mejorar así la función renal y evitar el desarrollo de ERC y enfermedad renal en etapa terminal durante el curso de la vida. ${ }^{11}$

\section{CONCLUSIONES}

La programación del desarrollo del riñón fetal impacta sobre las ECNT a través del efecto mediador del RCIU, del BP y la prematuridad. La mayoría de los factores maternos que impactan sobre el BP y la prematuridad no existen en forma aislada. Sus efectos sobre el desarrollo del riñón son muy relevantes para las mujeres en países en desarrollo, donde la prevalencia de recién nacidos de BP, PEG y PP es elevada.

Las recomendaciones presentadas coinciden con los Objetivos de Desarrollo Sostenible propuestos por las Naciones Unidas, para que disminuir la pobreza, mejorar la seguridad alimentaria, disminuir el embarazo adolescente, empoderar y educar a las niñas y a las mujeres, reducir las infecciones maternas y controlar las enfermedades crónicas que pueden reducir el riesgo de BP, PEG, parto prematuro, preeclampsia, diabetes gestacional y obesidad materna e infantil puedan lograr un impacto positivo en la salud renal de las generaciones futuras.

\section{Agradecimientos}

A la Dra. Adriana Iturzaeta, por su inestimable ayuda en la búsqueda bibliográfica y en la corrección del manuscrito.

\section{REFERENCIAS}

1. World Health Organization. Global Action Plan for the Prevention and Control of NCDs, 2013-2020. [Acceso: 6 de abril de 2020]. Disponible en: https:/ / www.who.int / publications/i/item/9789241506236

2. World Health Organization. Sustainable Development Goals 2015. [Acceso: 4 de marzo de 2020]. Disponible en: http:/ / sustainabledevelopment.un.org

3. White SL, Perkovic V, Cass A, Chang C, et al. Is low birth weight an antecedent of CKD in later life? A systematic review of observational studies. Am J Kidney Dis. 2009; 54(2):248-61.

4. Abi Khalil C, Travert F, Fetita S, Rouzet F, et al. Fetal exposure to maternal type 1 diabetes is associated with renal dysfunction at adult age. Diabetes. 2010;59(10):2631-6.

5. Lee YQ, Collins CE, Gordon A, Rae KM, et al. The relationship between maternal nutrition during pregnancy and offspring kidney structure and function in humans: A Systematic Review. Nutrients. 2018; 10(2):241.

6. Dorey EM, Pantaleon M, Weir KA, Moritz KM. Adverse prenatal environment and kidney development: implications for programing of adult disease. Reproduction. 2014; 147(6):R189-98.

7. Vieux R, Fresson J, Guillemin F, Hascoet J. Perinatal drug exposure and renal function in very preterm infants. Arch Dis Child Fetal Neonatal Ed. 2011; 96(4):F290-5.

8. Tzur S, Rosset S, Shemer R, Yudkovsky G, et al. Missense mutations in the APOL1 gene are highly associated with end stage kidney disease risk previously attributed to the MYH9 gene. Hum Genet. 2010; 128(3):345-50.

9. Hoy WE, Hughson MD, Bertram JF, Douglas-Denton R, et al. Nephron number, hypertension, renal disease, and renal failure. J Am Soc Nephrol. 2005; 16(9):2557-64.

10. Sutherland MR, Gubhaju L, Moore L, Kent A, et al. Accelerated maturation and abnormal morphology in the preterm neonatal kidney. J Am Soc Nephrol. 2011; 22(7):1365-74.

11. Fanos V, Gerosa C, Loddo C, Faa G. State of the art on kidney development: How nephron endowment at birth can shape our susceptibility to renal dysfunction later in life. Am J Perinatol. 2019; 36(S 02):S33-6.

12. LuyckxV, Brenner B. Birth weight, malnutrition and kidneyassociated outcomes-a global concern. Nat Rev Nephrol. 2015; 11(3):135-49.

13. Ingelfinger JR, Nuyt A-M. Impact of Fetal programming, birth weight, and infant feeding on later hypertension. J Clin Hypertens (Greenwich). 2012; 14(6):365-71.

14. Hughson M, Farris A 3rd, Douglas-Denton R, Hoy W, et al. Glomerular number and size in autopsy kidneys: The relationship to birth weight. Kidney Int. 2003; 63(6):2113-22.

15. Puelles V, Hoy W, Hughson M, Diouf B, et al. Glomerular number and size variability and risk for kidney disease. Curr Opin Nephrol Hypertens. 2011; 20(1):7-15.

16. Brenner BM, García DL, Anderson S. Glomeruli and blood pressure. Less of one, more the other? Am J Hypertens. 1988; 1(4 Pt 1):335-47.

17. CharltonJR,Springsteen $\mathrm{CH}$, Carmody JB. Nephron number and its determinants in early life: a primer. Pediatr Nephrol. 2014; 29(12):2299-308.

18. Schreuder MF. Safety in glomerular numbers. Pediatr Nephrol. 2012; 27(10):1881-7.

19. Paquette K, Fernandes RO, Xie LF, Cloutier A, et al. Kidney size, renal function, angiotensin peptides, and blood pressure in young adults born preterm. Hypertension. 2018; 72(4):918-28.

20. Ingelfinger JR, Woods LL. Perinatal programming, renal development, and adult renal function. Am J Hypertens. 2002; 15(2 Pt 2):46S $\square 9$.

21. Hoy WE, Bertram JF, Douglas-Denton R, Zimanyi M, et al. Nephron number, glomerular volume, renal disease and hypertension. Curr Opin Nephrol Hypertens. 2008; 17(3):25865.

22. Low Birth Weight and Nephron Number Working Group. The Impact of Kidney Development on the Life Course: A Consensus Document for Action. Nephron. 2017; 136(1):3-49.

23. Myrie SB, McKnight LL, Van Vliet BN, Bertolo RF. Low birth weight is associated with reduced nephron number and increased blood pressure in adulthood in a novel spontaneous intrauterine growth-restricted model in Yucatan miniature Swine. Neonatology. 2011; 100(4):380-6.

24. Boubred F, Laurent D, Buffat C, Tsimaratos M, et al. The magnitude of nephron number reduction mediates intrauterine growth-restriction-induced long term chronic renal disease in the rat. A comparative study in two experimental models. J Transl Med. 2016; 14(1):331.

25. Kett M, Denton K. Renal programming: cause for concern? Am J Physiol Regul Integr Comp Physiol. 2011;300(4):R791-803.

26. Doan T, Briffa J, Phillips A, Leemaqz S, et al. Epigenetic 
mechanisms involved in intrauterine growth restriction and aberrant kidney development and function. J Dev Orig Health Dis. 2020; 1-11.

27. Merlet-Bénichou C, Vilar J, Lelièvre-Pégorier M, Gilbert T. Role of retinoids in renal development: pathophysiological implication. Cur Opin Nephrol Hypertens. 1999; 8(1):39-43.

28. Wlodek M, Mibus A, Tan A, Siebel A, et al. Normal lactational environment restores nephron endowment and prevents hypertension after placental restriction in the rat. J Am Soc Nephrol. 2007; 18(6):1688-96.

29. Baldelomar EJ, Charlton JR, Beeman SC, Hann BD, et al. Phenotyping by magnetic resonance imaging nondestructively measures glomereular number and volume distribution in mice with and without nephron reduction. Kidney Int. 2016; 89(2):498-505.

30. RemuzziG,Benigni A, Remuzzi A.Mechanisms of progression and regression of renal lesions of chronic nephropathies and diabetes. J Clin Invest. 2006; 116(2):288-96.

31. Taal MW, Brenner BM. Predicting initiation and progression of chronic kidney disease developing renal risk scores. Kidney Int. 2006; 70(10):1694-705.

32. Tsuboi N, Kanzaki G, Koike K, Kawamura T, et al. Clinicopathological assessment of the nephron number. Clin Kidney J. 2014; 7(2):107-14.

33. Aisa MC, Cappuccini B, Barbati A, Orlacchio A, et al. Biochemical parameters of renal impairment/injury and surrogatemarkers of nephronnumberinintrauterine growthrestricted and preterm neonates at 30-40 days of postnatal corrected age. Pediatr Nephrol. 2016; 31(12):2277-87.

34. Cappuccini B, Torlone E, Ferri C, Arnone S, et al. Renal echo3D and microalbuminuria in children of diabetic mothers: a preliminary study. J Dev Orig Health Dis. 2013; 4(4):285-9.

35. Skálová S. The diagnostic role of urinary N-acetyl-B-D glucosaminidase (NAG) activity in the detection of renal tubular impairment. Acta Medica (Hradec Kralove). 2005; 48(2):75-80.

36. Rousian M, Verwoerd-Dikkeboom CM, Koning AH, Hop WC, et al. Early pregnancy volume measurements: validation of ultrasound techniques and new perspectives. BJOG. 2009; 116(2):278-85.

37. Brennan S, Schneider M, Watson D, Kandasamy Y, et al. The renal parenchyma-evaluation of a novel ultrasound measurement to assess fetal renal development: protocol for an observational longitudinal study. BMJ Open. 2017; 7(12):e019369.

38. Nusken E, Spencer L, Wohlfarth M, Lippach G, et al. Wholetranscript expression analysis identifies new candidate genes involved in renal tubular programming after uteroplacental insufficiency in rats. J Dev Orig Health Dis. 2015; 6(Suppl 2):S140.

39. Lurbe E, Wühl E. Hypertension in children and adolescent: New perspectives. New York: Springer; 2019 